\title{
Augmented Reality for Deaf Students: Can Mobile Devices Make It Possible?
}

\author{
Becky Sue Parton, Robert Hancock, and John Dawson \\ Southeastern Louisiana University
}

\begin{abstract}
Digital and real world events can be combined to create powerful learning opportunities for students, but time, tools, and expertise have been traditional barriers to teacher-created enhancements. This paper provides a rationale for using emerging, teacher-friendly tools, to merge real space and virtual space through video and 2D barcodes. The results of three pilot studies combine to illustrate the potential for using these tools. Results indicate that cell phones have the potential to facilitate augmented reality experiences for deaf students and adults.
\end{abstract}

Keywords: Augmented Reality, Mobile Devices, Accessibility, Deaf.

\section{Introduction}

According to Burik and Kelly [1], active learning has long been considered a proven method for increasing attention, motivation, and retention of concepts especially among deaf students. Although there are many definitions for interactive multimedia, Akhtar [2] described it as using many different media (print, audio, video, etc) to present more comprehensive information than any medium alone can, accommodating children with different learning styles, and employing interactivity to stimulate children to become active, motivated learners. Beyond the commercial and researcher-oriented instructional design projects, perhaps those with the most practical impact are the ones created by individual instructors. As the design tools become easier to use and more affordable, teachers can craft specially designed interactive software in a reasonable amount of time [3]. Every year more interactive material is developed by the teachers and that in turn has led to students participating in the design process themselves. For example, at the Laurent Clerc National Deaf Education Center, students collaborated to build an interactive video dictionary [4].

In taking the concept of interactive multimedia to an even higher plane, researchers have coined the term 'physical world hyperlinking' to capture the notion of connecting a tangible object to an on-line resource. The idea is to converge 'realspace' and 'mobilespace'. Tangible technology is a term that refers to directly linking computerbased activities with real-world physical objects and events $[5,6,7]$. When digital information is presented to users that enhances their real world surroundings the term Augmented Reality is often used. For example, popular applications for the iPhone such as Wikitude allow users to focus their phone camera on their surroundings and 
see data, like restaurant locations, layered on top of the image. For Deaf students, the augmented information could be sign translations.

The ultimate purpose of this paper is to explore how 2D barcodes and You Tube integrated digital video can be combined by a classroom teacher to provide augmented reality experiences accessible through cell phones for Deaf students. In order to provide a rationale for using those tools, it is necessary to look at a pilot study that focused on Global Positioning Satellite (GPS) technology.

\section{Augmented Reality Experience One: Using GPS}

A pilot set of materials were developed in late 2007 for the purpose of enhancing an on-site fieldtrip experience at Tiger Creek Wildlife Refuge with digital content. Tiger Creek is home to about forty big cats, most of whom have been rescued from abusive situations. The sanctuary provided the background stories for each tiger, and the lead author recorded video clips of a certified interpreter retelling the stories in American Sign Language (ASL). The video had to be captured and then compressed for web use - a total of 25 were created. Finally, a website was constructed to contain the videos, along with still photos, of each tiger. The research team then took GPS readings near each tiger exhibit area, recorded them in mapping software (Microsoft Streets and Trips), and coded the various flag points to launch web pages.

A group of seven high school students from the Jean Massieu Academy for the Deaf volunteered to participate in the pilot. In advance of the fieldtrip, a three hour training session was held for the purpose of showing the students how to use a micro laptop (a Sony Vaio UX) and GPS unit. At Tiger Creek each student had approximately forty-five minutes to locate an exhibit and launch the interpreted story.

The pilot project provided valuable insight into the concept of linking the digital and physical world. In written feedback and individual post-study interviews, all the students expressed positive comments regarding the value of the video component and the enjoyment level of the experience as a whole. For example one student said, "It's so awesome - just to have fun with the computer and enjoy seeing the real tigers". The researchers examined the screen capture footage from the laptop and determined that participants spent an average of 11 minutes and 8 seconds actively using the laptop to find flags, launch web pages, and watch video stories. The rest of the time the student was either watching the live tigers, taking photos/notes, or discussing events with peers or guides.

However, there were many limitations to the experience. First, from the perspective of the teachers who accompanied the group, the event was not duplicable. Specialized skills were required to make the videos, post the videos on websites, and code the GPS points - all of which was time consuming. In addition, the hardware was expensive and not readily available in the school. From the perspective of the research team, the system appeared cumbersome in regards to the GPS because a signal was often hard to obtain due to the tree coverage. The battery life of the micro laptop was also an issue. Thus a major finding of the study was the conflict of student satisfaction with the practical feasibility for teacher produced fieldtrips. 


\section{Augmented Reality Experience Two: Using 2D Barcodes}

Based up on the pilot study outlined above, the researchers set out to find an alternative approach that was less labor-intensive and not cost-prohibitive but equally enjoyable for students. A process that is currently in widespread use in Japan, and to a limited extent in other countries, is called 2D barcode camera phone scanning. Using this technology, a person walking around a Cineplex might notice movie posters that had a 2D barcode image and use his/her cell phone to take a picture of that image which would launch the trailer video. Free software is available to users who want to create their own QR codes at http://qrcode.kaywa.com. This technology appeared to be a viable option for hybrid fieldtrips, so two studies were designed: 1) a survey to find out if teachers had access cell phones capable of running the barcode software and 2) to determine the best hardware for launching the barcodes.

In order to read the images, users need a camera phone with the reader software installed. In late 2008, an on-line survey was sent to 547 K-12 educators. The educators had self-selected themselves into the group receiving the survey because of their previous use with Hotchalk learning environments, an ad-based alternative to Blackboard. One hundred and twenty three teachers completed the survey and all participants indicated that they owned a cell phone. Two questions were asked on the survey: 1) Can you view web pages from your cell phone? And 2) Does your cell phone have a built-in camera? These two features are required in order for barcode software to work, although not all cell phones capable of performing these two actions have barcode software available. Data revealed that $49 \%$ of the teachers had web viewing capability and $81 \%$ had photo taking capability on the phones. Although not part of the survey study, it should be noted that as each day passes, there are more phones on the market, such as Apple's iPhone that have this capability.

Beyond the issue of whether teachers have cell phones capable of reading 2D Barcodes, is the issue of how easy or difficult it is for people to activate the barcode launch mechanism. Therefore, the research team setup a second study. At a statelevel technology conference a booth was setup to test a participant's ability to retrieve the linked data from two encoded barcodes with two different cell phones. The sample size was thirty one people who voluntarily chose to participate, but three surveys were removed from the analysis because they were only partially completed.

The first barcode linked to a short URL, whereas the second barcode linked to a much longer URL thus requiring more bits of data to be encoded. The two cell phones used for the study were a Motorola $\mathrm{Q}$ with a 1.3 mega pixel camera and an AT\&T Tilt with a 3.0 mega pixel camera. Subjects tried to read both barcodes with both phones and then responded to a survey. They were asked to mark whether a successful launch of the linked information occurred for each combination and then to rate the ease of use from one to seven with seven being the friendliest. Factors influencing ease of use may have included the number of tries (i.e. length of time) it took for the camera to recognize the code, the steadiness required to read the codes, the distance that the user had to maintain from the code, and the phone's interface for maneuvering between the code reading screen and the web browser.

Three patterns emerge from the data: 1) All the participants were successful in being able to use the barcode reader to launch content, 2) A cell phone with better resolution (i.e. more megapixels) was easier to use, and 3) Barcodes that were encoded 
were more complex data were harder to read with low resolution cameras, but the difference became less noticeable with the higher resolution camera. Specifically, only $35.7 \%$ of the subjects rated the $1.3 \mathrm{mp}$ camera easy to use (a score of 6 or 7 ) in regards to the complex URL; whereas, $100 \%$ of the subjects rated the $3.0 \mathrm{mp}$ camera easy to use (a score of 6 or 7) in regards to the complex URL. Based upon these findings, the research team decided to re-design the field trip experience.

Although 2D barcodes could have been linked to the web pages created previously during the Tiger Creek pilot, there was a concern about the embedded video being universally playable across multiple types of phones. Additionally, the research team wanted to continue making the concept of merged realties more attainable for the average teacher so a decision was made to link directly to You tube videos rather than websites. Many user-friendly camcorders are capable of creating and posting you tube videos without any video editing skills or equipment such as the Flip camcorder and even the iPhone itself. For the Tiger Creek presentations, however, the researchers still created the You tube videos by mixing still photos and the previously captured interpreted story videos. The resulting videos were uploaded to You tube and the barcodes were generated. A working barcode for the tiger, Mowgli, can be found at: http://www.lifted-up.org/awe/TigerWeb/barcodes.htm. For readers who do not have a barcode reader, the image is also directly hyperlinked to the video. In actual use, the barcodes are printed and distributed to participants. The response to the revised materials was positive enough to warrant construction of a set of cards for the New Orleans Zoo where a formal study is anticipated this year.

Students want interactive, multimedia experiences. Until recently, teachers have been unable in many cases to develop that type of enhanced material because of the skills, equipment, and time required. This paper has outlined how a once complex hybrid fieldtrip can now be created by a teacher with limited access to a computer.

\section{References}

1. Burik, L., Kelly, W.: Active learning through technology-creating a technology- infused environment to engage Deaf students in the learning process. Paper Presented at the Technology and Disabled Persons Conference, Las Angeles, CA (2003)

2. Akhtar, A.: A study of interactive media for Deaf learners in post 16 education. Paper Presented at the Technology and Education of the Deaf Symposium, Rochester, NY (June 2003)

3. Rawlings, L. (n.d.): Clicker4 and More - multimedia and multiple support. Unpublished Document Retrieved November 10 (2003), http: / / education.qld.gov . au

4. Stifter, R.: Integrating technology and literacy: Digital video dictionary. Paper Presented at the Technology and Education of the Deaf Symposium, Rochester, NY (2001)

5. Chipman, G., Druin, A., Beer, D., Fails, J., Guha, M., Simms, S.: A case study of tangible flags: a collaborative technology to enhance field trips. Paper Presented at the 5th International Conference for Interactive Design and Children (IDC), Tampere, Finland (2006)

6. Price, S.: A representation approach to conceptualizing tangible learning environments. Paper Presented at the 2nd International Conference on Tangible and Embedded Interaction (TEI), Bonn, Germany (2008)

7. Sung, J., Levisohn, A., Song, J., Tomassetti, B., Mazalek, A.: Shadow box: an interactive learning toy for children. Paper Presented at the International Workshop on Digital Game and Intelligent Toy Enhanced Learning (DIGITEL), Jhongli City, Taiwan (2007) 